\title{
Three new species of Boesenbergia (Zingiberaceae) from Thailand and Lao P.D.R.
}

\author{
J.D. Mood ${ }^{1}, \dagger$ J.F. Veldkamp², T. Mandákováa ${ }^{3}$ L.M. Prince ${ }^{4}$ \& H.J. de Boer ${ }^{5}$ \\ ${ }^{1}$ Lyon Arboretum, University of Hawaii, 3860 Manoa Road, \\ Honolulu, Hi 96822, USA. \\ boesenbergia@gmail.com \\ 2 Naturalis Biodiversity Center, Section Botany, P.O. Box 9517, \\ 2300 RA Leiden, The Netherlands \\ ${ }^{3}$ CEITEC - Central European Institute of Technology, and Faculty of Science, \\ Masaryk University, Kamenice 5, 62500 Brno, Czech Republic \\ ${ }^{4}$ The Field Museum, Department of Botany, 1400 S Lake Shore Dr., \\ Chicago, IL 60605, USA \\ ${ }^{5}$ Natural History Museum, University of Oslo, Postboks 1172, \\ Blindern, 0318 Oslo, Norway
}

\begin{abstract}
Boesenbergia bella Mood \& L.M.Prince, B. phengklaii Mood \& Suksathan, and B. putiana Mood \& L.M.Prince are described with photographs and a comparative table. The description of Boesenbergia petiolata Sirirugsa is revised to include morphology not previously noted. Molecular phylogenetic analyses of the relevant taxa using plastid and nuclear DNA sequence data are provided.
\end{abstract}

Keywords. Boesenbergia bella, B. petiolata, B. phengklaii, B. putiana, chromosome counts, nrITS, molecular phylogeny, trnK, Vietnam

\section{Introduction}

Beginning in 2010, a taxonomic update of Boesenbergia (Zingiberaceae) was initiated for the Flora of Thailand project. The research model was based on a species by species review of those currently recorded in Thailand. The study of each included the protologue, types and all similar specimens, history, field and ex situ data, results of molecular phylogenetic analyses, and photography. The first to be studied was Boesenbergia longiflora (Wall.) Kuntze which resulted in the description of five new species (Mood et al., 2013). Since then, several other species have been reviewed and published (Mood et al., 2014a,b, 2016a,b). In this study, Boesenbergia petiolata Sirirugsa is reviewed.

The first known specimen of this species, Pierre s.n. P02203112 (P), was collected in Cochinchine (Vietnam) by the French botanist, Jean Baptiste Louis Pierre (1833-1905), Director of the Jardin Botanique in Saigon from 1865-1877. Sometime during his tenure, this small, petite plant was found and later deposited at the Muséum National d'Histore Naturelle in Paris without annotation or date. In 1917, a similar ginger with well-preserved floral parts was collected in Giá Rai, Bạc Liêu Province, Cochinchine, Chevalier 36.458 (P). The first recorded specimen in Thailand was from 
Saraburi Province in 1928, Phraisurind 2419 (BK), but it was not until 1987 that this and similar specimens were studied in detail and recognised as being of an undescribed species (Sirirugsa, 1987). The specimen selected as the type, Maxwell 74-631 (holotype BK; isotype L), was collected in 1974 at Sahm Lan (now Namtok Sam Lan National Park), Saraburi Province, central Thailand. In addition to the types, approximately 26 other collections have been found that were either annotated as Boesenbergia petiolata or were unidentified but were similar to $B$. petiolata.

Over the course of field work (2013-2015), locations in northern, central and eastern parts of Thailand were visited which corresponded to areas documented on the specimens. Many of the original locations were difficult to locate and some had been lost through development. In total, eight locations were visited that provided sufficient materials for study. In addition to these, two living accessions were added from the Lao P.D.R.

On comparing these ex situ living collections over several years the following observations were made: the first annual growth, flowering cycle, and die-back into dormancy were not calendar concurrent for all nine collections; the vegetative growth habit varied from tightly clumping (tufted) plants to ones with wider-spaced stems; and the leaf and floral characters were not uniform. On the other hand, similarities included: inflorescence shape/size and the same combination of colours on the labellum to include a yellowish tint (especially during senescence).

From the field and ex situ observations, it became clear that six of the ten collections differed considerably from the type of Boesenbergia petiolata and were most likely of three distinct, undescribed species. Molecular phylogenetic analyses were carried out to investigate evolutionary relationships among these accessions.

\section{Materials and methods}

Herbarium specimens, silica-dried leaf samples, and living plant materials (rhizomes) were collected on site from 2012-2015. Living materials were grown in Hawaii, USA, for comparison and long-term observation. A few collections were also grown at Queen Sirikit Botanic Gardens, Thailand. Ecological data were recorded at each site. Photographic documentation of plants and flowers was accomplished both in the field and ex situ. The Boesenbergia petiolata type material along with specimens with similar morphology were studied and compared at BK, BKF, QBG and SING. High resolution digital photos were taken of each specimen for later reference. Photographs from earlier visits to AAU, C, CMU, E, K, L, P, PNU, and VNM were also used for comparison.

\section{Molecular phylogeny}

Leaf tissue samples were obtained from living materials in the field, or in the case of M96P82 and M3471 from ex situ plants. Selected GenBank sequences deposited as part of previous studies by our research group were combined with data from the current study. A list of included samples (with associated GenBank accession numbers) is provided in Appendix 1. 
Methods are consistent with prior publications from our research group. DNA extraction from silica-dried samples was done using the CTAB extraction protocol (Kress et al., 2002). DNA amplification, sequencing, and analytical methods follow Mood et al. (2013). The nuclear ribosomal ITS (nrITS) region was amplified using the plant specific primers developed by Prince (2010): 18S-F (CGATTGAATGGTCCGGTGAAG) and 26S-R (AGGACGCTTCTACAGACTACAA). The plastid trnK region was amplified in two parts, the first using either 1F (CTCAACGGTAGAGTACTCG; Manos \& Steele, 1997) and mIR (CGTTTCACAAGTACTGAACTA) or 1F and 1235R (TATACTAATACCCCACYCC). The second half was amplified using mIF (GTTCAGTACTTGTGAAACGTT; Kress et al., 2002) and 2R (AACTAGTCGGATGGAGTAG; Steele \& Vilgalys, 1994). Amplicons were bidirectionally sequenced on an ABI Genetic Analyzer at The Field Museum (Chicago, Illinois, USA), and verified and edited in Sequencher v4.9 (Gene Codes Corporation, Ann Arbor, Michigan, USA). The resulting contigs were aligned in Se-Al (Rambaut, 1996) and exported as Nexus files.

Phylogenetic analyses were conducted under both maximum parsimony and likelihood criteria in PAUP* (version 4.0b10; Swofford, 2002). Exploratory heuristic searches of the family-wide data matrices were limited to maximum parsimony to assess monophyly of the ingroup. Parameters for these exploratory analyses were 10,000 random addition replicates, saving 10 trees per replicate. Similarly, exploratory bootstrap analyses of the large data matrix were limited to 100,000 fast swap replicates. Alignment of sequences across the family were challenging for the ITS region. No data was excluded and gaps were treated as missing data.

After monophyly was confirmed, most of the outgroup taxa and many of the non-ingroup Boesenbergia taxa were removed, the data realigned, and more elaborate heuristic search methods were employed (maximum parsimony with 1000 random addition replicates, saving all shortest trees; maximum likelihood with 10 random addition replicates). No data were excluded and gaps were treated as missing data here as well. Likelihood model selection was conducted in ModelTest 3.7 (Posada \& Crandall, 1998). If the Hierarchical Likelihood Ratio Test (hLRT; Felsenstein, 1981) and the Akaike Information Criterion (AIC; Akaike, 1974) differed in the model selected, analyses were run under each model. Branch support was estimated using heuristic search parameters in a parsimony bootstrap (BS; Felsenstein, 1985) in PAUP* and posterior probabilities (PP) via Bayesian analysis in MrBayes 3.2 (Ronquist et al., 2011; conducted via the CIPRES portal, Miller et al., 2010). Bootstrap analyses used 1000 random addition replicates with 100 pseudoreplicates (hold 1 tree, TBR branch swapping, save 10 trees per replicate). Bayesian analyses ran for 5 million generations and were repeated three times, burn-in set to $<0.01$ standard deviation of the splits frequencies. Results from all three runs were used to generate posterior probabilities. Only posterior probabilities $\geq 0.95$ are shown. Parsimony analysis, likelihood model parameter selection and analysis results, and Bayesian run details are provided in Appendix 2. 
Chromosome preparations and counting.

Actively growing, young roots were harvested from cultivated plants, and subsequently pre-treated with ice-cold water for 12 hours, fixed in ethanol/acetic acid $(3: 1, \mathrm{v} / \mathrm{v})$ fixative for 24 hours at $4^{\circ} \mathrm{C}$ and stored at $-20^{\circ} \mathrm{C}$ until further use. Selected root tips were rinsed in distilled water (twice for 5 minutes) and citrate buffer ( $10 \mathrm{mM}$ sodium citrate, $\mathrm{pH} 4.8$; twice for 5 minutes), and digested in $0.3 \%(\mathrm{w} / \mathrm{v})$ cellulase, cytohelicase and pectolyase (all Sigma-Aldrich, St Louis, MO, USA) in citrate buffer at $37^{\circ} \mathrm{C}$ for 90 minutes. After digestion, individual root tips were dissected on a microscope slide in approximately $10 \mu \mathrm{l}$ acetic acid and covered with a cover slip. The cell material was then spread evenly using tapping, thumb pressing and gentle flame-heating. Finally, the slide was quick frozen in liquid nitrogen and the cover slip flicked off with a razor blade. Slides were fixed in ethanol-acetic acid (3:1) and air-dried. Chromosomes were counterstained with $2 \mu \mathrm{g} / \mathrm{ml}$ DAPI in Vectashield (Vector Laboratories, Peterborough, UK). Preparations were analysed and photographed using a Zeiss Axioimager Z2 epifluorescence microscope with a CoolCube camera (MetaSystems). At least 20 mitotic chromosome spreads were counted from each accession analysed.

\section{Results and discussion}

Exploratory genus-wide analyses of ITS sequences identified a modestly-supported Boesenbergia petiolata sensu lato clade (73\% fast BS; results not shown) containing nine ingroup sequences. The $\operatorname{trnK}$ data analyses identified a well-supported Boesenbergia petiolata sensu lato clade (with 99\% fast BS; results not shown), but not all accessions were included in the supported clade. A combined ITS + trnK analysis however identified a ten sample, monophyletic Boesenbergia petiolata sensu lato clade with modest support ( $89 \%$ fast BS; results not shown).

Parsimony and likelihood analyses of sequences in the target clade (plus a group of place holder samples) resulted in trees of similar in group topology. Phylograms from analyses of the ITS matrix alone (Appendix 3) identified a monophyletic Boesenbergia petiolata clade ( $84 \%$ BS, $1.00 \mathrm{PP})$, with resolution of three internal clades, a $B$. petiolata sensu stricto clade (100\% BS, 1.00 PP), a novel clade A (63\% $\mathrm{BS}, 0.78 \mathrm{PP})$, and a novel clade B (99\% BS, $1.00 \mathrm{PP})$. Results of the $\operatorname{trnK}$ matrix alone (Appendix 3) identified a monophyletic Boesenbergia petiolata sensu stricto clade (99\% BS, $1.00 \mathrm{PP})$ plus a monophyletic clade of morphologically similar accessions, but generally provided lower branch support and less resolution.

Phylogenies from the combined ITS $+\operatorname{trn} K$ analyses (Fig. 1) were fully resolved and well supported, regardless of analytical method. Topologies within the ingroup were similar across all trees. The best likelihood tree (AIC criterion) is shown in Figure 1 with parsimony BS and Bayesian PP branch values indicated. For marker-specific phylograms of ITS and trnK per analysis method see Appendix 3. A well supported (91\% BS, 1.00 PP) Boesenbergia petiolata sensu lato clade was identified, including a monophyletic $B$. petiolata sensu stricto $(100 \% \mathrm{BS}, 1.00 \mathrm{PP})$, the novel clade A $(87 \%$ $\mathrm{BS}, 1.00 \mathrm{PP})$, and the novel clade B (99\% BS, $1.00 \mathrm{PP})$. At the base of clades A and B (99\% BS, $1.00 \mathrm{PP})$, a clade $\mathrm{C}$, consisting of one accession, is sister to A and $\mathrm{B}$. 


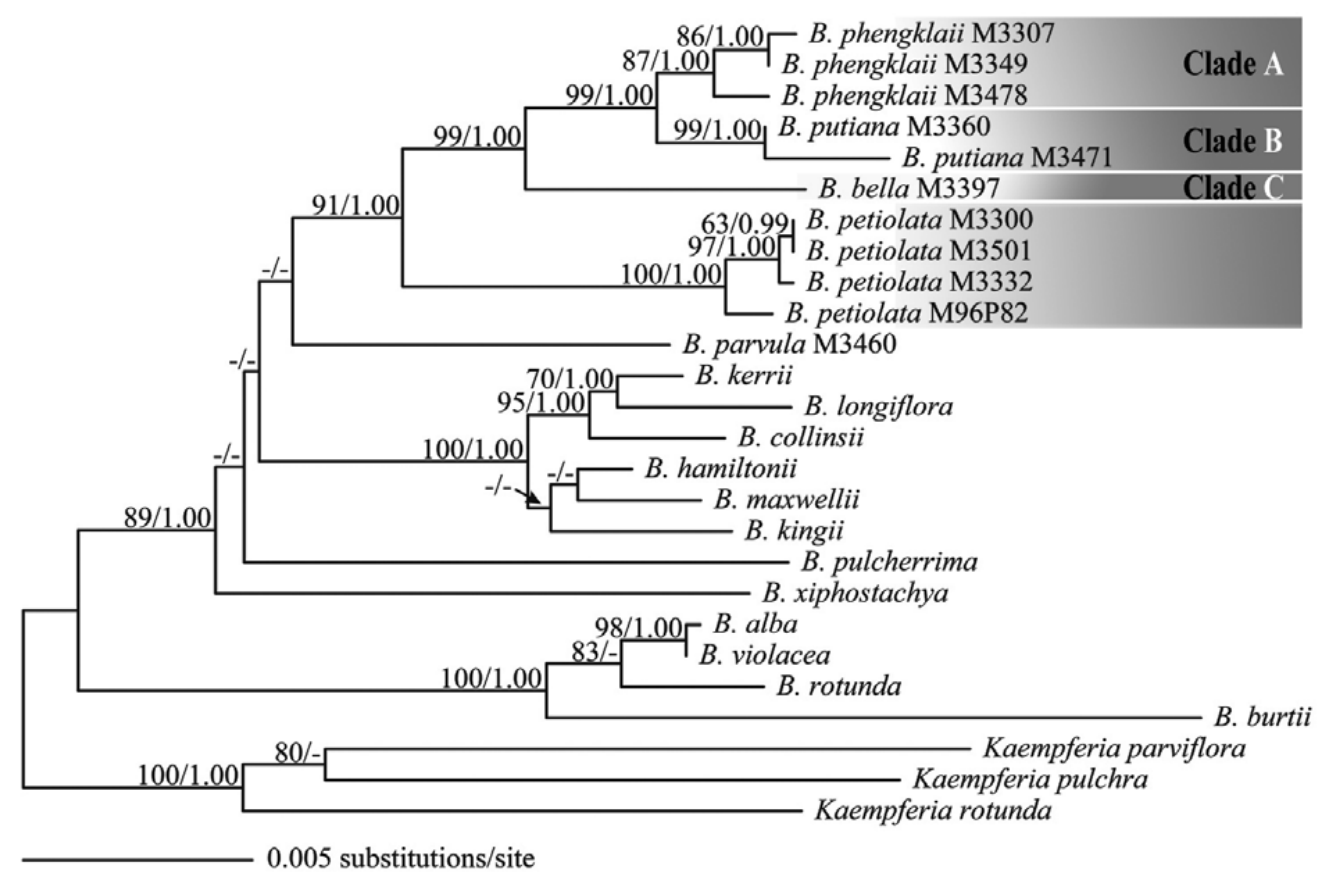

Fig. 1. Maximum likelihood phylogram of combined ITS and trnK data analysis for Boesenbergia petiolata and closely related taxa based on the General Time Reversible $(\mathrm{GTR}+\mathrm{I}+\mathrm{G})$ model. Branch support values are BS/PP. See text for details.

Based on the results of the molecular phylogeny a reassessment of the morphological characters of all Boesenbergia petiolata collections was made. This study confirmed the presence of a morphologically consistent monophyletic Boesenbergia petiolata sensu stricto clade as well as a clade of species related to Boesenbergia petiolata that are each morphologically coherent and can be distinguished from other Boesenbergia clades by their flower shape and coloration. As a result, three new species are described along with an updated circumscription of Boesenbergia petiolata. The new species are Boesenbergia bella Mood \& L.M.Prince, B. phengklaii Mood \& Suksathan and B. putiana Mood \& L.M.Prince. Boesenbergia phengklaii Mood \& Suksathan corresponds to clade A, B. putiana Mood \& L.M.Prince to clade B and $B$. bella Mood \& L.M.Prince to clade C (Fig. 1).

\section{Taxonomy}

Three new species are described along with an updated circumscription of Boesenbergia petiolata. A comparison of morphological characters for the four species is presented in Table 1. 
Table 1. Comparison of $B$. petiolata to the three newly described species.

\begin{tabular}{|c|c|c|c|c|}
\hline $\begin{array}{l}\text { Character/ } \\
\text { Species }\end{array}$ & $\begin{array}{l}\text { Boesenbergia } \\
\text { bella }\end{array}$ & $\begin{array}{l}\text { Boesenbergia } \\
\text { petiolata }\end{array}$ & $\begin{array}{l}\text { Boesenbergia } \\
\text { phengklaii }\end{array}$ & $\begin{array}{l}\text { Boesenbergia } \\
\text { putiana }\end{array}$ \\
\hline Type locality & Lao P.D.R. & Thailand & Thailand & Thailand \\
\hline $\begin{array}{l}\text { Soil } \\
\text { derivation }\end{array}$ & Sandstone & Sandstone/Granite & $\begin{array}{l}\text { Sandstone/Lime- } \\
\text { stone }\end{array}$ & Shale/Limestone \\
\hline Forest type & Deciduous & Deciduous & Deciduous & Semi-deciduous \\
\hline Elevation & c. $250 \mathrm{~m}$ & $50-700 \mathrm{~m}$ & $300-700 \mathrm{~m}$ & c. $300 \mathrm{~m}$ \\
\hline $\begin{array}{l}\text { Growth } \\
\text { habit }\end{array}$ & Clumping & Spreading & Spreading & Spreading \\
\hline $\begin{array}{l}\text { Plant height } \\
\text { incl. leaves }\end{array}$ & to $40 \mathrm{~cm}$ & to $50 \mathrm{~cm}$ & to $55 \mathrm{~cm}$ & to $60 \mathrm{~cm}$ \\
\hline Sheaths & $\begin{array}{l}\text { Red, white } \\
\text { spotted/ } \\
\text { corrugate/ } \\
\text { pubescent }\end{array}$ & $\begin{array}{l}\text { Green or reddish/ } \\
\text { striate/glabrous }\end{array}$ & $\begin{array}{l}\text { Green, red } \\
\text { spotted, or solid } \\
\text { red/striate/ } \\
\text { glabrous }\end{array}$ & $\begin{array}{l}\text { Light green/ } \\
\text { corrugate/ } \\
\text { glabrous }\end{array}$ \\
\hline Ligule & $\begin{array}{l}0.5 \mathrm{~cm} / \text { apex } \\
\text { round }\end{array}$ & $\begin{array}{l}1.5 \mathrm{~cm} / \text { apex } \\
\text { triangular }\end{array}$ & $\begin{array}{l}\text { to } 0.8 \mathrm{~cm} / \text { apex } \\
\text { round }\end{array}$ & $\begin{array}{l}\text { to } 1 \mathrm{~cm} / \text { apex } \\
\text { triangular }\end{array}$ \\
\hline Lamina size & $\begin{array}{l}5.5-14 \times \\
13-25 \mathrm{~cm}\end{array}$ & $4.5-8 \times 18-23 \mathrm{~cm}$ & $5-8 \times 12-22 \mathrm{~cm}$ & $\begin{array}{l}9-14 \times 15-27 \\
\mathrm{~cm}\end{array}$ \\
\hline $\begin{array}{l}\text { Lamina } \\
\text { colour } \\
\text { Dorsal/ } \\
\text { Ventral }\end{array}$ & $\begin{array}{l}\text { Tri-coloured/ } \\
\text { wine red }\end{array}$ & $\begin{array}{l}\text { Light to dark } \\
\text { green/silvery green } \\
\text { or purple }\end{array}$ & $\begin{array}{l}\text { Dark green/ } \\
\text { silvery green or } \\
\text { purple }\end{array}$ & $\begin{array}{l}\text { Dark green/ } \\
\text { silvery green }\end{array}$ \\
\hline No. of bracts & $14-18$ & $6-14$ & $13-16$ & c. 12 \\
\hline $\begin{array}{l}\text { Labellum } \\
\text { shape/ } \\
\text { flattened } \\
\text { size }\end{array}$ & $2.2 \times 2.0 \mathrm{~cm}$ & $\begin{array}{l}2.5-2.8 \times 2.0-2.5 \\
\mathrm{~cm}\end{array}$ & $2.8 \times 2.5 \mathrm{~cm}$ & $3.2 \times 2.6 \mathrm{~cm}$ \\
\hline $\begin{array}{l}\text { Labellum } \\
\text { colour \& } \\
\text { pattern }\end{array}$ & $\begin{array}{l}\text { White, spotted } \\
\text { red throat, red } \\
\text { streaked } \\
\text { margin, apex } \\
\text { violet }\end{array}$ & $\begin{array}{l}\text { White, spotted red, } \\
\text { throat to apex, } \\
\text { margins yellow, } \\
\text { apex red spots }\end{array}$ & $\begin{array}{l}\text { White, spotted } \\
\text { throat, margins } \\
\text { mostly white, } \\
\text { apex solid red }\end{array}$ & $\begin{array}{l}\text { White, streaked } \\
\text { red throat, } \\
\text { margins light } \\
\text { yellow, apex } \\
\text { striped }\end{array}$ \\
\hline $\begin{array}{l}\text { Asexual } \\
\text { propagules }\end{array}$ & $\begin{array}{l}\text { Bulbils (ex } \\
\text { situ) }\end{array}$ & None observed & Bulbils (ex situ) & None observed \\
\hline
\end{tabular}


Boesenbergia bella Mood \& L.M.Prince, sp. nov.

Boesenbergiae petiolatae (Wall.) Kuntze affinis, foliis c. $6 \mathrm{~cm}$ latis multicoloratis, labelli apice violaceo bilobato differt. TYPE: Lao P.D.R., north of Pakse, in hills east of $15^{\circ} 22.236^{\prime} \mathrm{N} 105^{\circ} 48.789^{\prime} \mathrm{E}$, c. $248 \mathrm{~m}$ asl, collected as Paprataro s.n., cultivated in Hawaii, USA as J.D. Mood 3397, vouchered on 1 October 2016 as J.D. Mood 16P07 and voucher selected as type (holotype BKF; isotype AAU). (Fig. 2, 6-8)

Deciduous, perennial herb up to $40 \mathrm{~cm}$ tall, multiple stems, c. $1 \mathrm{~cm}$ apart. Rhizome branched, length variable to $10 \mathrm{~cm}, \mathrm{c} .0 .5 \mathrm{~cm}$ diam., externally orange-brown, internally light orange, tuberous roots numerous, to $10 \mathrm{~cm}$ long, c. $0.5 \mathrm{~cm}$ diam., externally and internally white, persistent and enlarging over several years, apex tapered, surface with numerous short roots. Stems $10-15$ per clump, to $10 \mathrm{~cm}$ long, c. $1 \mathrm{~cm}$ diam., white, few hairs, striate, leafless sheaths 1-2, c. $8 \mathrm{~cm}$ long, to $3 \mathrm{~cm}$ wide (flattened), light red, greenish toward the apex, mostly glabrous, few hairs on margins, corrugate, green spots between the ridges, apex rounded, minutely unguiculate. Leaves 2-3, upper two nearly opposite; leaf sheaths 6-8 cm long, 2-3 cm wide at the base (flattened), corrugate, red throughout with white spots, pubescent, short white hairs between the ridges; ligule bilobed, auriculate, each lobe to $0.5 \mathrm{~cm}$ long, semi-translucent, red spotted, with long white hairs throughout, prominent red veins, apex acute, cucullate, margins white; petiole c. $4 \mathrm{~cm}$ long, c. $0.5 \mathrm{~cm}$ wide, green, red spotted, with scattered few hairs, ridged, channelled; lamina ovate to elliptical, 13-25 $\times 5.5-14 \mathrm{~cm}$, base rounded to acute, oblique, apex shortly acuminate, adaxially tri-coloured, midrib dark red, then a dark green, feathered area either side with two, wide, silver bands, margins dark green, glabrous, primary veins raised, reticulate between the primary and secondary veins, abaxially dark burgundy red, iridescent, glabrous, midrib green, veins sunken. Inflorescence terminal on a leafy stem, basal portion clasped between the leaf sheaths, $1 / 4$ to $1 / 2$ exposed; peduncle to $0.5 \mathrm{~cm}$ long, $0.8 \mathrm{~cm}$ diam., white, pubescent, ridged; spike to c. $11 \mathrm{~cm}$ long, c. $1.2 \mathrm{~cm}$ wide; bracts $14-18$, lanceolate, to $4.5 \mathrm{~cm}$ long, to 1 $\mathrm{cm}$ wide at the base (flattened), white, numerous long white hairs, apex acute, bracts distichously arranged, compressed together in two ranks on one side, the rachis exposed on the non-flowering side, rachis $0.8 \mathrm{~cm}$ wide, light green, glabrous; bracteole ovate, to $4.7 \mathrm{~cm}$ long, to $1.5 \mathrm{~cm}$ (flattened), larger than the bract, white, glabrous, apex falsely pungent. Flowers c. $4.5 \mathrm{~cm}$ long, all facing outward from the bract; calyx tubular, c. $0.8 \mathrm{~cm}$ long, c. $0.3 \mathrm{~cm}$ diam., translucent white, sparse hairs, apex bifid, with unilateral incision; floral tube c. $2.5 \mathrm{~cm}$ long, $0.2 \mathrm{~cm}$ diam., white, glabrous; dorsal corolla lobe oblong, c. $1.6 \times 0.5 \mathrm{~cm}$, white, glabrous, three-veined, apex slightly cucullate, sometimes revolute; lateral corolla lobes oblong, c. $1.4 \times 0.4 \mathrm{~cm}$, white, glabrous, three-veined, apex slightly cucullate; androecial cup c. $0.2 \times 0.2 \mathrm{~cm}$, initially oriented c. $45^{\circ}$ to the floral tube on opening, throat with few hairs either side; labellum saccate, nearly orbicular (flattened), $2.2 \times 2.0 \mathrm{~cm}$, white, midrib marked with red spots to $1 / 2$ the length, then darker red bands spreading outward $1 / 4$ length, then a broad violet band to the apex, margin white or light yellow, turning darker yellow toward maturity, crisped, crenulate, exterior glabrous; lateral staminodes ovate, c. $1 \times 0.5 \mathrm{~cm}$, white, turning slightly yellow at maturity, internally and externally glabrous, apices acute, slightly 
revolute. Stamen c. $1 \mathrm{~cm}$ long, filament c. $0.3 \times 0.1 \mathrm{~cm}$, channelled, white, anther c. $0.8 \mathrm{~cm}$, white, thecae c. $1 \mathrm{~mm}$ wide when mature, dehiscent the full length, connective tissue with glandular hairs, apex truncate; pollen white. Ovary trilocular, style filiform, c. $4 \mathrm{~cm}$ long, white, stigma funnel-shaped, white, ostiole rounded, margin tri-lobed, non-ciliate; epigynous glands $2 \times 1 \mathrm{~mm}$, yellowish, segmented in two sections, top half diameter smaller than lower half. Fruit not seen. Bulbils cylindrical, c. $2 \times 0.5$ $\mathrm{cm}$, green, sericeous, occurring at the base of the inflorescence.

Distribution. Known only from Champasak Province, Lao P.D.R.

Ecology. Found in lightly shaded locations in deciduous forest on sandstone derived soils rich in organic matter. Associated species: Boesenbergia rotunda (L.) Mansf., Gagnepainia sp., Kaempferia champasakensis Picheans. \& Koonterm, K. gigantiphylla Picheans. \& Koonterm, and Zingiber pellitum Gagnep.

Phenology. In cultivation, flowering from July - mid-October.

Etymology. Named for its beauty.

Chromosome count. $2 \mathrm{n}=18$.

Notes. This species came to the first author's attention at Chatuchak Market, Bangkok, Thailand where it was sold by a local Thai vendor. The small, tufted plant presented one of the most striking examples of a Boesenbergia ever seen. Later it was found to have been collected in the Lao P.D.R.

Boesenbergia petiolata Sirirugsa, Nordic J. Bot. 7: 423 (1987). TYPE: Thailand, Saraburi Province, Nam Tok Sam Lan National Park, Maxwell 74-631, 29 June 1974 (holotype BK; isotypes AAU, L). (Fig. 3, 6-8)

Deciduous, perennial herb to $50 \mathrm{~cm}$ tall, multiple stems, 1-5 $\mathrm{cm}$ apart. Perennating organ a rhizome, branched, expanding linearly, length variable, c. $1 \mathrm{~cm}$ diam., externally orange-brown, internally yellowish, tuberous roots numerous, persistent and enlarging over several years, $3-12 \mathrm{~cm}$ long, $0.5-0.8 \mathrm{~cm}$ diam., externally and internally white, apex tapered, surface with numerous short roots. Stems 10-15 per clump, to $25 \mathrm{~cm}$ long, 0.8-1.2 cm diam., light green to dark green, glabrous, leafless sheaths $0-1$, c. 4 $\mathrm{cm}$ long, $1 \mathrm{~cm}$ wide (flattened), striate, green, glabrous, apex acute to rounded. Leaves 4-5, alternate, upper two nearly opposite; leaf sheaths 7-13 cm long, striate, green, glabrous; ligule bilobed, each lobe to $1.5 \mathrm{~cm}$ long, translucent white or pink, glabrous, veined, apex obtuse; petiole $2.5-8 \mathrm{~cm}$ long, $0.2-0.4 \mathrm{~cm}$ wide, green, glabrous; lamina ovate to lanceolate, asymmetrical, $8-23 \times 4.5-8 \mathrm{~cm}$, base cordate, sometimes oblique, apex acuminate, adaxially light green to dark green, glossy, glabrous, primary veins raised, lightly reticulate, abaxially light silvery green, glabrous, veins sunken. 

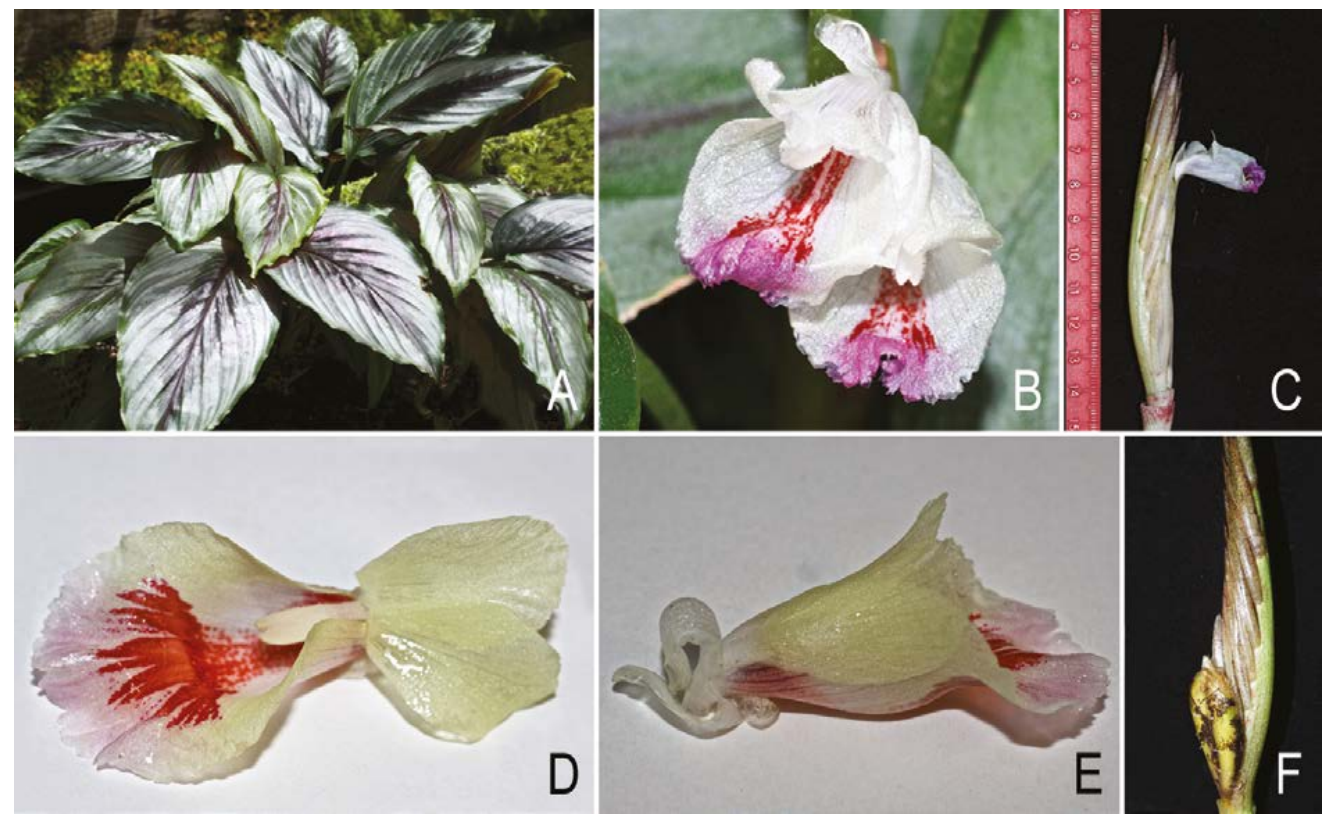

Fig. 2. Boesenbergia bella Mood \& L.M.Prince. A. Plant ex situ. B. Flowers. C. Inflorescence. D. Opened young flower. E. Flower in profile. F. Bulbils at the inflorescence base. All from M3397. (Photos: J. Mood).

Inflorescence terminal, distichous, basal portion clasped between the leaf sheaths, $1 / 4$ to $1 / 2$ exposed; peduncle c. $1 \mathrm{~cm}$ long, $0.5 \mathrm{~cm}$ diam., green, glabrous; spike c. $12 \mathrm{~cm}$ long, c. $1 \mathrm{~cm}$ wide; bracts 6-14, lanceolate, to $4 \mathrm{~cm}$ long, to $0.8 \mathrm{~cm}$ wide at the base (flattened), white with light green in upper half, glabrous, apex falsely pungent, bracts compressed together in two ranks on one side, the rachis exposed on the non-flowering side; bracteole lanceolate, to $4 \mathrm{~cm}$ long, to $0.8 \mathrm{~cm}$ wide (flattened), identical to the bract, white with green toward apex, glabrous, apex falsely pungent. Flowers c. $4 \mathrm{~cm}$ long, all facing outward from the bract, tilted slightly downward; calyx tubular, c. 0.5 cm long, c. $0.3 \mathrm{~cm}$ diam., translucent white, glabrous, apex trifid; floral tube 2-2.8 $\mathrm{cm}$ long, $0.1 \mathrm{~cm}$ diam., white, glabrous; dorsal corolla lobe oblong to obovate, c. 1.3 $\times 0.5 \mathrm{~cm}$, white, glabrous, three-veined, apex slightly cucullate, sometimes revolute; lateral corolla lobes oblong, c. $1 \times 0.3 \mathrm{~cm}$, white, glabrous, three-veined, apex slightly cucullate; androecial cup c. $0.2 \times 0.2 \mathrm{~cm}$, orientated c. $90^{\circ}$ to the floral tube, throat glabrous; labellum deeply saccate, nearly orbicular (flattened), 2.5-2.8 $\times 2.0-2.5 \mathrm{~cm}$, white, throat marked with two red spots, more red spots continue along all the veins outward, short of the apex, there longitudinal red stripes or spots in a broad patch reaching almost to the margins, margins slightly revolute, light yellow, becoming darker with age, slightly crisped, apex yellow, crisped, slightly crenulate, externally covered with a few, short, glandular hairs; lateral staminodes obovate, c. $1.3 \times 0.9$ $\mathrm{cm}$, white, internally and externally glabrous, apices slightly revolute. Stamen c. $1 \mathrm{~cm}$ long, filament c. $4 \times 0.2 \mathrm{~cm}$, channelled, yellowish white, anther c. $0.8 \mathrm{~cm}$ long, white, 
thecae c. $1 \mathrm{~mm}$ wide when mature, dehiscent the full length, dorsally covered with glandular hairs, apex slightly indented; pollen white. Ovary trilocular, style filiform, c. $3.8 \mathrm{~cm}$ long, white, stigma funnel-shaped, white, ostiole round, few cilia; epigynous glands $2 \times 1 \mathrm{~mm}$, yellowish. Fruit not seen.

Distribution. Central and eastern Thailand, Lao P.D.R., Vietnam.

Ecology. Semi-deciduous forest, normally on sandstone derived soils.

Phenology. In cultivation, flowering from July - mid-October.

Etymology. Named for its long petiole.

Chromosome count. $2 \mathrm{n}=18$.

Additional specimens examined. LAO P.D.R. Champasak: exact location data lost, Mood M96P82 (AAU, BKF).

THAILAND. Uttaradit: Nam Pat, Phu Soi Dao National Park, 680 m, 7 Aug 2013, Newman 2620 (QBG). Sukhothai: Khao Luang, 13 Sep 1994, Niyomdham 3850 (BKF); Ram Kham Haeng National Park, Khiri Mat, Khao Luang, 700 m, 11 Aug 2012, Middleton et al. 5563 (BKF). Phitsanulok: Thung Salaeng Luang, 500 m, 19 Jul 1966, Larsen 463 (AAU, BKF); Thung Salaeng Luang, 400 m, 24 Jul 1966, Larsen 837 (AAU, BKF); Thung Salaeng Luang National Park, Bahn Mung, 100 m, 9 Aug 2008, Sreepoowiang K44 (BKF, PNU); Thung Salaeng Luang, c. $250 \mathrm{~m}$ asl, Aug 2012, Vatcharakorn s.n., M3300 (BKF); Thung Salaeng Luang, 245 m asl, 24 Jul 2013, Mood \& Vatcharakorn 3332 (BKF); Ban Chom Phu, Noen Maprang, 28 Jul 2001, Maknoi 158 (QBG); Tham Pha Tha Phon Non-hunting Area, Noen Maprang, 7 Sep 2011, Norsaengsri \& Insea 8149 (QBG). Khon Kaen: Phaphuang Cave, Phu Pha Man National Park, 460 m, 14 Aug 2012, Norsaengsri 9883 (QBG); Phu Pha Man National Park, 488 m, 16 Aug 2012, Norsaengsri 9971 (QBG). Kamphaeng Phet: Klong Lan Waterfall, 6 Aug 2003, Maknoi 452 (QBG). Sakon Nakhon: Phu Phan National Park, 290 m, 31 Jul 1999, Newman 950 (E). Saraburi: Hin Lap, 20 Aug 1929, Put 2419 (BK); Kao Ngob, 21 Aug 1945, Din 10 (BKF); Nam Tok Sam Lan, 76 m asl, 9 Aug 2015, Mood \& Harwood 3501 (BKF). Nakhon Nayok: Eastern part, Khao Yai National Park, 300 m, 10 Aug 1968, Larsen 3252 (AAU, BKF). Surat Thani: Ban Thakhun Dist., Khlong Saeng Wildlife Sanctuary, 90 m, 6 Sept 2008, Middleton et al. 4306 (E).

VIETNAM. No location or date, Pierre s.n. [P02203112] (P). Bạc Liêu: Giá Rai District, Sep 1917, Chevalier 36458 (P). Đak Nong: Draysap Waterfall, 380 m asl, 27 Jul 2009, Trần 161 (E, SING, VNMd).

Notes. The description above is based on Boesenbergia petiolata sensu stricto with thin, light green foliage, cordate leaf bases, and yellowish flowers with light red markings. The measurements are based on living material of M3501 (type locality) and cultivated material of M96P82 (Lao P.D.R.). It should be recognised that at several locations cited, especially in Phitsanulok Province (Chat Trakan, Noen Maprang, and Thung Salaeng Luang), the populations have slightly different character states. In these populations, the leaves are more robust with mostly rounded leaf bases, the abaxial leaf surfaces 


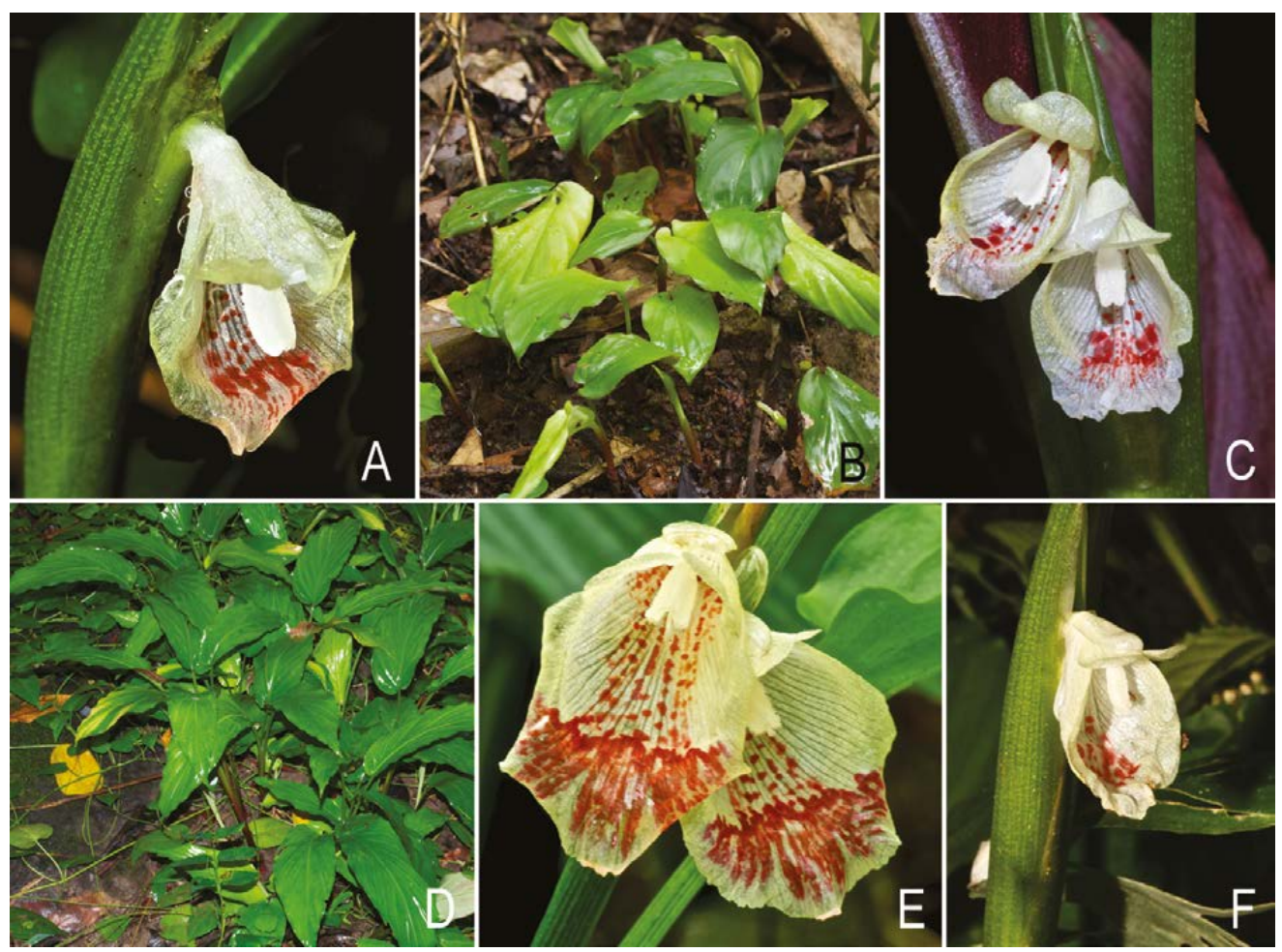

Fig. 3. Boesenbergia petiolata Sirirugsa. A. Flower at type locality. B. Young plants at type locality. C. Old and young flower showing abaxial leaf colour. D. Plants of M3300. E. Old and young flowers of M96P82 (Lao P.D.R.) F. Flower of Trần 161 (Vietnam). B from M3501; C \& D from M3300; E from M96P82; F from Trần 161. (Photos: A, R. Pooma, BKF; B-E, J. Mood; F, H.Đ. Trần.)

have red pigmentation, the flowers have less yellow tinting overall, the labellum has a darker red centre, and the labellum apex has violet colouring (Fig. 2 C-D). Nonetheless, based on the molecular phylogenetic results, these samples (M3300, M3332) show a very close affinity to plants from the type locality as represented by $M 3501$.

Boesenbergia phengklaii Mood, sp. nov.

Boesenbergiae petiolatae (Wall.) Kuntze affinis, caulibus vaginisque maculose ad solide rubris, labello atrorubro apice violaceo differt. TYPE: Thailand, Chiang Mai Province, Doi Suthep foothills, $18^{\circ} 48.063^{\prime} \mathrm{N} 98^{\circ} 56.026^{\prime} \mathrm{E}$, c. $635 \mathrm{~m}$ asl, collected as Mood \& Vatcharakorn 3349, cultivated in Hawaii, USA as M3349, vouchered on 1 October 2016 as Mood $16 \mathrm{PO}$ and voucher selected as type (holotype BKF; isotype AAU). (Fig. 4, 6-8)

Deciduous, perennial herb to $55 \mathrm{~cm}$ tall, multiple stems, c. $1 \mathrm{~cm}$ apart. Perennating organ a rhizome, expanding linearly, length variable, $0.5-1.5 \mathrm{~cm}$ diam., externally 
brown, internally white, tuberous roots few, persistent, to $14 \mathrm{~cm}$ long, c. $0.3 \mathrm{~cm}$ diam., externally and internally white, apex a spheroid, c. $3 \mathrm{~cm}$ long, $1 \mathrm{~cm}$ diam., surface with few roots. Stems c. 15 per clump, to $30 \mathrm{~cm}$ long, c. $0.8 \mathrm{~cm}$ diam., green, glabrous, leafless sheaths 1-2, c. $7 \mathrm{~cm}$ long, striate, green spotted with red to a darker and solid red, glabrous, apex rounded to $0.8 \mathrm{~cm}$ wide. Leaves $4-5$, alternate, upper two nearly opposite; leaf sheaths 14-15 cm long, striate, green or spotted red to solid red, glabrous; ligule bilobed, each lobe $0.2-0.8 \mathrm{~cm}$ long, translucent green or spotted red, glabrous, apex rounded; petiole $3-6 \mathrm{~cm}$ long, $0.2-0.3 \mathrm{~cm}$ wide, green or red spotted, glabrous; lamina ovate, asymmetrical, $12-22 \times 5-8 \mathrm{~cm}$, base slightly cordate, or rounded to attenuate, sometimes oblique, apex acuminate, adaxially dark, glossy green, glabrous, primary and secondary veins prominently raised, reticulate, abaxially light silvery green, sometimes dark reddish-purple, glabrous, completely covered with minute, round bumps, silky to touch. Inflorescence terminal on a leafy shoot, basal portion clasped between the leaf sheaths, $1 / 4$ to $1 / 2$ exposed; peduncle c. $1 \mathrm{~cm} \mathrm{long,} 0.4 \mathrm{~cm}$ diam., white, glabrous; spike c. $9 \mathrm{~cm}$ long, c. $1 \mathrm{~cm}$ wide; bracts 13-16, lanceolate, 2.5$3.5 \mathrm{~cm}$ long, 0.3-0.4 cm wide at the base (flattened), white with light green in upper half, glabrous, covered with small gland-like markings, apex falsely pungent, bracts compressed together in two ranks on one side, the rachis exposed on the non-flowering side; bracteole lanceolate, $2.5-3.2 \mathrm{~cm}$ (flattened), slightly smaller than the bract, light green, glabrous, apex falsely pungent. Flowers c. $4.5 \mathrm{~cm}$ long, variable in size based on plant age, all facing outward from the bract, tilted slightly downward; calyx tubular, c. $0.6 \mathrm{~cm}$ long, translucent white, glabrous, apex bifid; floral tube c. $3.8 \mathrm{~cm}$ long, 0.2 cm diam., white, glabrous; dorsal corolla lobe ovate, c. $1.5 \times 0.4 \mathrm{~cm}$, white, glabrous, three-veined, apex slightly cucullate, sometimes revolute; lateral corolla lobes ovate, c. $1.7 \times 0.3 \mathrm{~cm}$, white, glabrous, three-veined, apex slightly cucullate, quickly revolute; androecial cup c. $0.2 \times 0.2 \mathrm{~cm}$, orientated c. $90^{\circ}$ to the floral tube, throat glabrous; labellum deeply saccate, broadly elliptical (flattened), c. $2.8 \times 2.5 \mathrm{~cm}$, white, throat marked with red, maculate red pattern extending outward on either side of the centre, ending at $3 / 4$ the length, then forming a broad rectangular area, white in the centre with a red margin, then red stripes to near the apex, apex interspersed with violet, margins crenulate, revolute c. $2 \mathrm{~mm}$ wide near the androecial tube, broadening to c. $1 \mathrm{~cm}$ at the apex, as the flower matures, the white colour yellows, especially along the margins, externally covered with short, glandular hairs; lateral staminodes obovate, c. $1.5 \times 1$ $\mathrm{cm}$, white, internally glabrous, externally with short, glandular hairs, apices slightly revolute. Stamen c. $1.5 \mathrm{~cm}$ long, white, glandular hairs along the backside, full length, filament c. $4 \times 1.5 \mathrm{~mm}$, channelled, white, anther c. $0.8 \mathrm{~cm}$, white, thecae c. $1 \mathrm{~mm}$ wide when mature, dehiscent the full length, apex slightly indented; pollen white. Ovary trilocular, style filiform, c. $5.3 \mathrm{~cm}$ long, white, stigma rounded, white, ostiole round, non-ciliate; epigynous glands $2 \times 1 \mathrm{~mm}$, yellowish. Fruit not seen.

Distribution. Currently known only from locations in Chiang Mai, Lampang and Phrae Provinces, northern Thailand. 

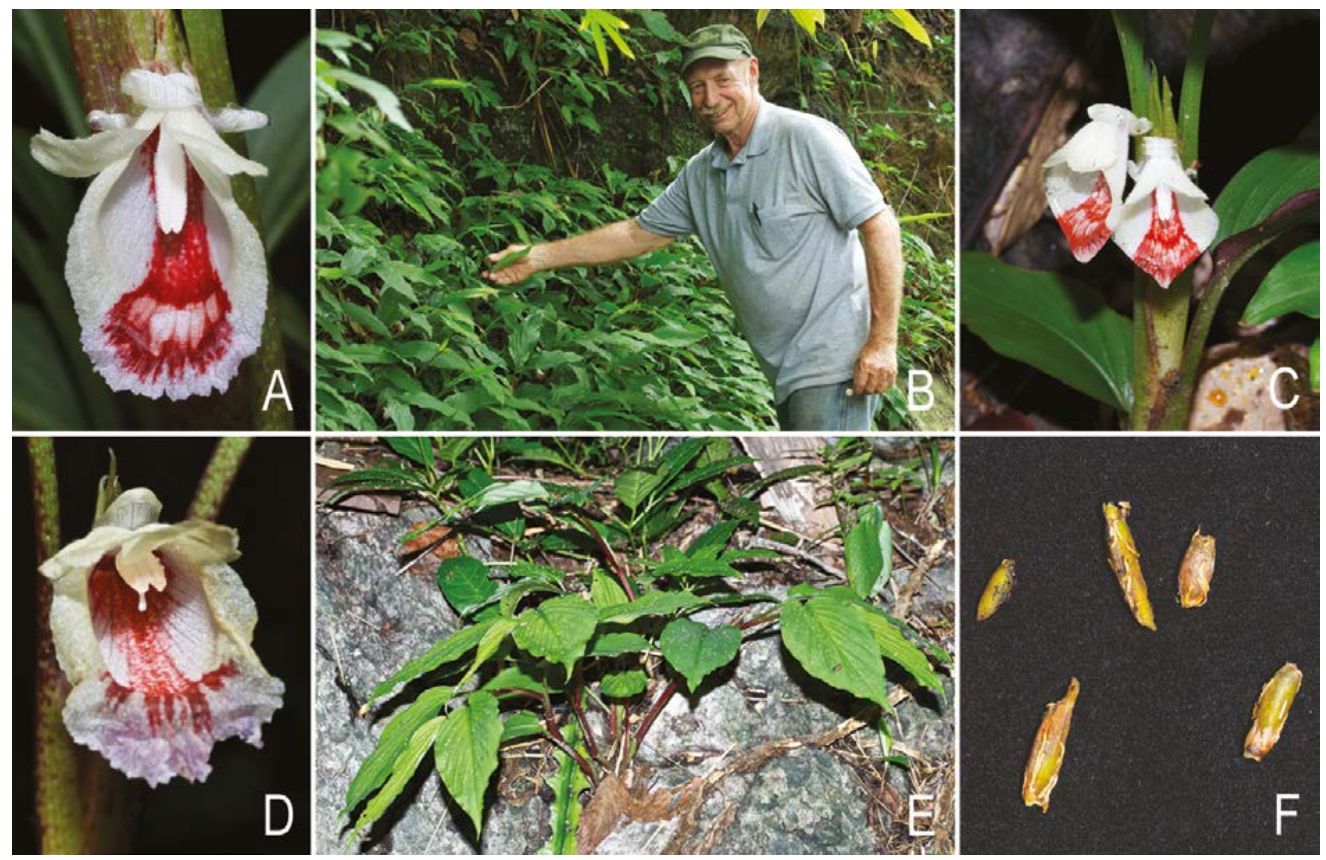

Fig. 4. Boesenbergia phengklaii Mood \& Suksathan. A. Young flower. B. First author with plants at Doi Suthep. C. Flowers at mid-day with margins turned under. D. Old flower with thecae spread and style released. E. Plants showing long, semi-succulent stems. F. Bulbils (c. $1 \mathrm{~cm}$ long) found on old plants going dormant. A-C from M3349; D- F from M3478. (Photos: J. Mood)

Ecology. Found in semi-deciduous forest on well-drained, sloped areas with light shade, often on rock surfaces. The Chiang Mai populations grow on fairly deep soil with high organic matter, while the Phrae plants grow in crevices with humus on large limestone outcrops.

Phenology. In cultivation, flowering occurs July through to early October.

Etymology. Named for Dr Chamlong Phengklai (BKF), one of the first collectors of this new species.

\section{Chromosome count. $2 \mathrm{n}=18$.}

Additional specimens examined. THAILAND: Chiang Mai: Doi Inthanon National Park, c. 550 m, 20 Jul 1988, Phengklai et al. 6631 (BKF); Doi Inthanon National Park, c. 650 m, 21 Jul 1988, Phengklai et al. 6725 (BKF); Doi Sutep, 850 m, 3 Jul 1958, Larsen 3866 (C); Muang, Doi Suthep National Park, Palaht Temple area, 740 m, 7 Aug 1993, Phuakam 45 (CMU, K); Unknown location/collector, 1 Jun 1932, BKF091304 (BKF); Muang, Doi Suthep National Park, east side, 500 m, 18 Jul 1987, Maxwell 87-690 (BKF, CMU); Doi Inthanon National Park, along Mae Klang River (RS-4), 570 m, 3 Aug 1988, Tamura T-60386 (BK); Doi Suthep 
National Park, $18^{\circ} 38.063^{\prime} \mathrm{N} 98^{\circ} 56.026^{\prime} \mathrm{E}, 633 \mathrm{~m}$ asl, $25 \mathrm{Jul} 2013$, Mood \& Vatcharakorn 3307 (BKF). Lampang: Rahng Chat, Doi Kuhn Dahn National Park, 400 m, 29 Jul 1994, Maxwell 94-818 (BKF). Phrae: Phukao Hin Pakarang, Pha Klong cave, near 1809.153'N 9959.079'E, c. $305 \mathrm{~m}$ asl, Aug 2015, Mood \& Suksathan 3478 (BKF, QBG).

Notes. The measurements given in the description are based on the living, cultivated material of M3349, the parent plant from which the type specimen was collected. Although all specimens of Mood \& Suksathan 3478 are similar to the type, this collection sports several differences. The plants of this population have long, semisucculent stems with widely-spaced leaves, while the type tends to have shorter internodes with fewer leaves which occur closer to the terminus. Also, the flowers of the variant have a slightly different red colour pattern on the labellum with more intense violet at the margins and apex. Bulbils were observed on ex situ plants of this collection. They are cylindrical, c. $1.8 \times 0.4 \mathrm{~cm}$, light yellowish green or dark green, covered with a few, short, and white roots. They are produced under the leaf sheaths near the nodes, breaking through as they mature (Fig. 4F). Bulbils were not observed in the wild. Whether they form in the natural environment has yet to be determined.

\section{Boesenbergia putiana Mood \& L.M.Prince, sp. nov.}

Boesenbergiae petiolatae affinis, planta maiore ad $60 \mathrm{~cm}$ longa caespitosa, labello atrorubre striato, maiore ad $3.2 \mathrm{~cm}$ lato differt. TYPE: Thailand, Mae Hong Son Province, Mueang District, near Tam Pla Pha Suea cave, 19²5.915'N 9759.871'E, c. $275 \mathrm{~m}$ asl, collected as Mood \& Vatcharakorn 3360, cultivated in Hawaii, USA as M3360, vouchered on 1 October 2016 as Mood $16 P 09$ and voucher selected as type (holotype BKF; isotype AAU). (Fig. 5-8)

Deciduous, perennial herb to $60 \mathrm{~cm}$ tall, robust, multiple stems, c. $1 \mathrm{~cm}$ apart. Perennating organ a rhizome expanding linearly, length variable, $0.5-1.5 \mathrm{~cm}$ diam., externally orange-brown, internally white, tuberous roots numerous, persistent and enlarging over several years, to $16 \mathrm{~cm}$ long, c. $1 \mathrm{~cm}$ diam., externally and internally white, apex sometimes a spheroid, surface with few short roots. Stems 15-20 or more per clump, to $20 \mathrm{~cm}$ long, c. $2 \mathrm{~cm}$ diam., light green, glabrous, leafless sheaths 1-2, broadly lanceolate, to $11 \times 3 \mathrm{~cm}$, corrugate, light green, glabrous, margins slightly revolute in upper half, apex deflexed, acute. Leaves 4-5, alternate, upper two nearly opposite; leaf sheaths 12-14 cm long, corrugate, light green, glabrous, margins deflexed outward; ligule bilobed, to $1 \mathrm{~cm}$ long, triangular, green, glabrous; petiole 4-6 cm long, $0.4 \mathrm{~cm}$ wide, deeply channelled, green, glabrous; lamina ovate, c. $15 \times$ $9 \mathrm{~cm}$ (lower), c. $27 \times 14 \mathrm{~cm}$ (upper), base truncate, apex acuminate, adaxially dark, glossy green, glabrous, primary veins prominently raised, abaxially light silvery green, red tinged on lower two leaves, glabrous, completely covered with minute, round bumps, silky to the touch. Inflorescence terminal on a leafy shoot, basal portion clasped between the leaf sheaths, $1 / 4$ exposed; peduncle c. $1 \mathrm{~cm}$ long, to $0.8 \mathrm{~cm}$ diam., white, glabrous; spike to $13 \mathrm{~cm}$ long, 1-2.2 cm wide; bracts distichous, lanceolate, c. 12 , to $6 \mathrm{~cm}$ long, c. $1 \mathrm{~cm}$ wide at the base (flattened), white with dark green in 


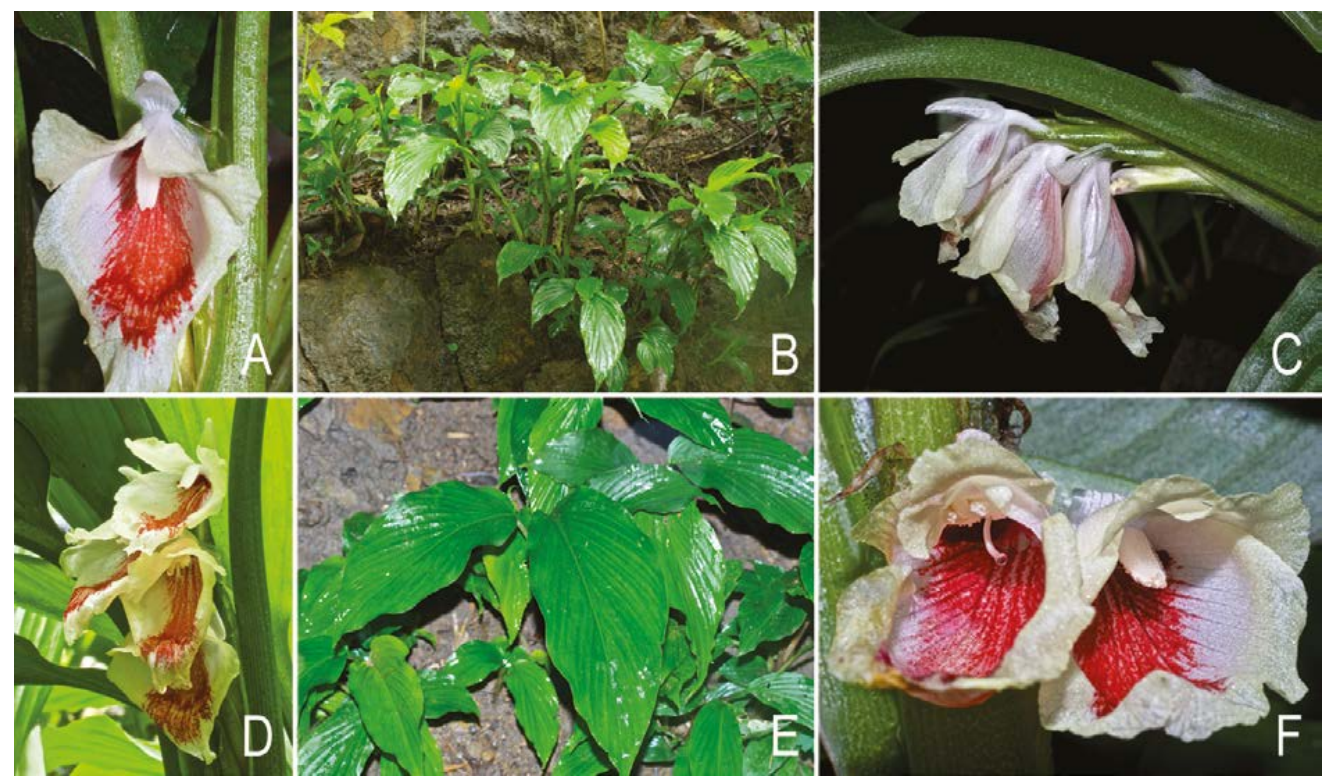

Fig. 5. Boesenbergia putiana Mood \& L.M.Prince. A. Young flower. B. Plants. C. Flowers in profile. D. Flowers showing new and old. E. Leaf close-up. F. Old flower with thecae rotated and nectar drop on stigma (left), young flower with thecae closed and stigma not released. All from M3360. (Photos: J. Mood)

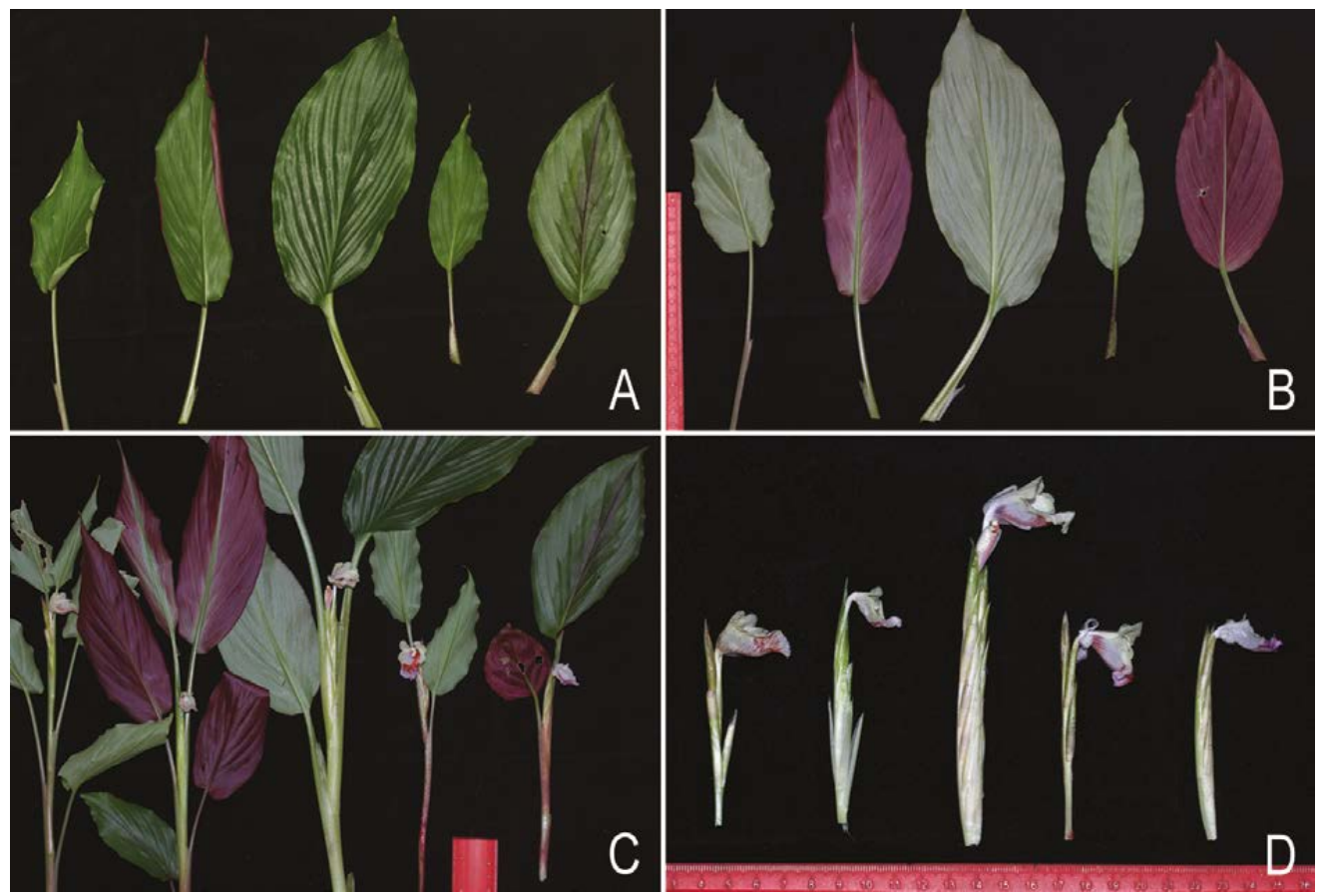

Fig. 6. Left to right in each picture-Boesenbergia petiolata Sirirugsa (M96P82), B petiolata Sirirugsa (M3300), B. putiana Mood \& L.M.Prince (M3360), B. phengklaii Mood \& Suksathan (M3349), B. bella Mood \& L.M.Prince (M3397). A. Leaf adaxial surface. B. Leaf abaxial surface. C. Plant with inflorescence and flower. D. Inflorescence (unsheathed) and flower. (Photos: J. Mood) 
upper third, glabrous, apex falsely pungent, bracts tightly compressed together in two ranks on the flowering side, angled at $45^{\circ}$ to the rachis, rachis exposed on the nonflowering side, c. $1 \mathrm{~cm}$ diam. at the base, light green, glabrous; bracteole lanceolate, to $6 \mathrm{~cm}$ long, c. $1 \mathrm{~cm}$ wide at the base, light green, glabrous, apex falsely pungent, completely clasping the floral tube. Flowers c. 4-5 cm long, variable in size based on plant age, all facing outward from the bract, tilted slightly downward; calyx tubular, c. $1 \mathrm{~cm}$ long, translucent white, glabrous, apex bifid; floral tube c. $3 \mathrm{~cm}$ long, $0.2 \mathrm{~cm}$ diam., white, glabrous; dorsal corolla lobe oblong, c. $1.8 \times 0.6 \mathrm{~cm}$, white, glabrous, five-veined, apex slightly cucullate; lateral corolla lobes lanceolate, c. $1.7 \times 0.5 \mathrm{~cm}$, white, glabrous, three-veined, apex slightly cucullate; androecial cup c. $0.3 \times 0.3 \mathrm{~cm}$, orientated c. $90^{\circ}$ to the floral tube, throat glabrous; labellum deeply saccate, broadly elliptical (flattened), c. $3.2 \times 2.6 \mathrm{~cm}$, white, throat marked with red lines, broadening outward, fan-like onto the deflexed portion, ending at $3 / 4$ the length, from there to the apex white, margins mostly entire, wavy, sometimes notched, apex crinkled, as the flower matures the margins turn yellowish, externally covered with short, glandular hairs; lateral staminodes obovate, c. $2.0 \times 1.1 \mathrm{~cm}$, white, internally glabrous, externally with short, glandular hairs, apices slightly revolute. Stamen c. $1.2 \mathrm{~cm}$ long, white, few glandular hairs along the backside, filament c. $4 \times 2 \mathrm{~mm}$, deeply channelled, white, anther c. $8 \mathrm{~mm}$ long, $4 \mathrm{~mm}$ wide, white, thecae c. $1 \mathrm{~mm}$ wide when mature, dehiscent the full length, apex slightly indented; pollen white. Ovary trilocular, style filiform, c. $1.5 \mathrm{~cm}$ long, white, stigma rounded, white, ostiole rounded, non-ciliate; epigynous glands c. $2 \times 1 \mathrm{~mm}$, yellowish. Fruit not seen.

Distribution. Known only from Mae Hong Son Province, Thailand.

Ecology. Found growing in small populations under medium shade in evergreen forests on (what appears to be) calcareous shale or limestone of the Thong Pha Phum group.

Phenology. In cultivation, flowering from July - September.

Etymology. This species is named for Put Phraisurind, the Thai botanical assistant of A.F.G. Kerr. During his tenure (1920-1932), Put collected 4548 numbers of Thai plants which were deposited at ABD, BK, BKF, BM, E, K, L, MO, NY, and perhaps other herbaria.

Chromosome count. $2 \mathrm{n}=18$.

Additional specimens examined: THAILAND: Mae Hong Son: No exact locality, Wongnaks.n. [QBG 20130908] (QBG); Pang Mapha, Panyachan s.n. [QBG 20140685A] (QBG); Mueang, Ban Huay Pha, Tham Pha Daeng, 260 m, 1 Aug 2013, Newman et al. 2583 (QBG); Na Pla Chad, Tham Pla-Namtok Pha Suea National Park, 322 m, 11 Sep 2013, Lakoet 0394 (QBG).

Notes. The measurements in the description are based on the living, cultivated material of $M 3360$, the parent plant from which the type specimen was collected. 


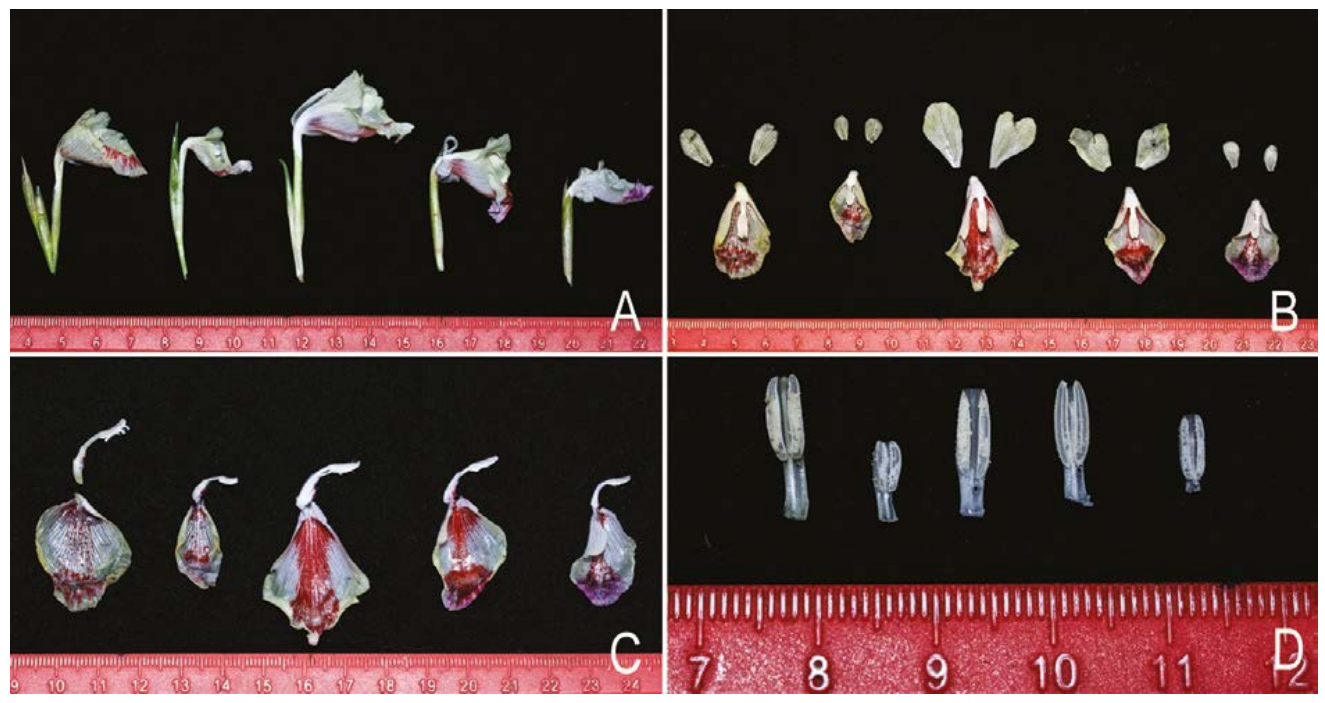

Fig. 7. Left to right in each picture-Boesenbergia petiolata Sirirugsa (M96P82), B. petiolata Sirirugsa (M3300), B. putiana Mood \& L.M.Prince (M3360), B. phengklaii Mood \& Suksathan (M3349), B. bella Mood \& L.M.Prince (M3397). A. Flower and bract in profile. B. Labellum and lateral staminodes. C. Labellum with anther. D. Anther. (Photos: J. Mood)
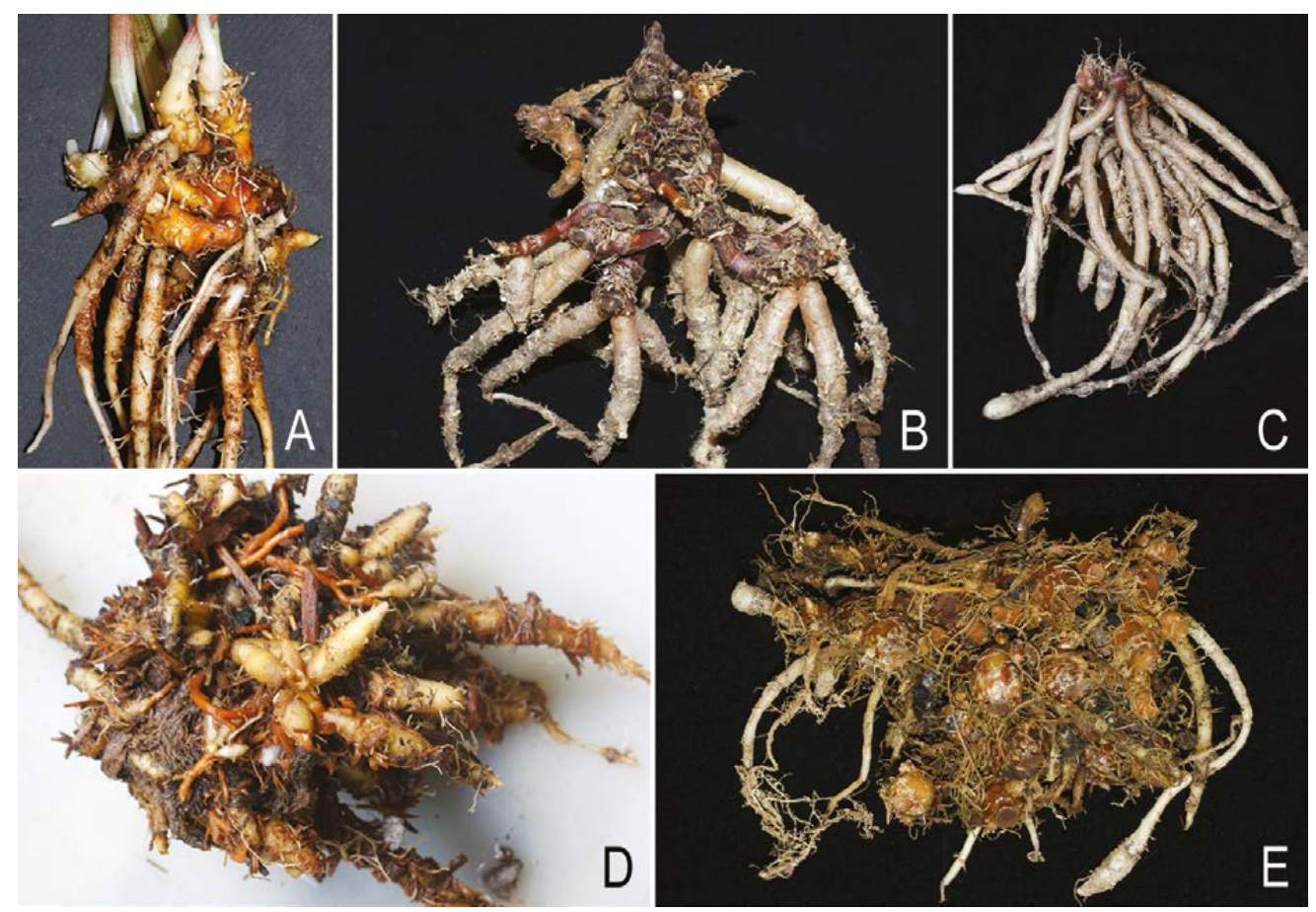

Fig. 8. Rhizomes. A. Boesenbergia petiolata Sirirugsa. B. B. petiolata Sirirugsa. C. B. putiana Mood \& L.M.Prince. D. B. bella Mood \& L.M.Prince. E. B. phengklaii Mood \& Suksathan. A from M96P82; B from M3300; C from M3360; D from M3397; E from M3349. (Photos: J. Mood) 


\section{Conclusions}

As a result of the morphological and molecular phylogenetic findings presented here, three new species are described as distinct from Boesenbergia petiolata. All four species form a clade with close affinity based on a common ancestor. The chromosome number of $2 \mathrm{n}=18$ is unique to the clade, whereas the sister species, Boesenbergia parvula (Wall.) Kuntze and numerous other Thai species are $2 \mathrm{n}=20$.

ACKNOWLEDGEMENTS. We would like to especially thank P. Vatcharakorn (Chanthaburi), R. Harwood (BKF), and staff of the Queen Sirikit Botanical Garden for field assistance and donations of their collections of ginger rhizomes. We also thank the Plant Quarantine office in Bangkok for phytosanitary certificates and the USDA APHIS in Honolulu, Hi. for import; The Field Museum, Chicago, Il USA (F) for use of Pritzker DNA Laboratory facilities; and Linda Ann Vorobik (USA) for the plate compositions.

\section{References}

Akaike, H. (1974). A new look at the statistical model identification. IEEE Trans. Automatic Control 19: 716-723.

Felsenstein, J. (1981). Evolutionary trees from DNA sequences: a maximum likelihood approach. J. Molec. Evol. 17: 368-376.

Felsenstein, J. (1985). Confidence intervals on phylogenies: an approach using the bootstrap. Evolution 39: 783-791.

Kress, W.J., Prince, L.M. \& Williams, K.J. (2002). The phylogeny and a new classification of the gingers (Zingiberaceae): Evidence from molecular data. Amer. J. Bot. 89: 1682 $-1696$.

Manos, P.S. \& Steele, K. P. (1997). Phylogenetic analyses of "higher" Hamamelididae based on plastid sequence data. Amer. J. Bot. 84: 1407-1419.

Miller, M.A., Pfeiffer, W. \& Schwartz, T. (2010). Creating the CIPRES Science Gateway for inference of large phylogenetic trees. In: Proceedings of the Gateway Computing Environments Workshop (GCE), pp. 1-8. New Orleans, LA: IEEE.

Mood, J.D., Prince, L.M., Veldkamp, J.F. \& Dey, S. (2013). The history and identity of Boesenbergia longiflora (Zingiberaceae) and descriptions of five related new taxa. Gard. Bull. Singapore 65(1): 47-95.

Mood, J.D., Veldkamp, J.F. \& Prince, L.M. (2014a). A new species and a new record of Boesenbergia (Zingiberaceae) for Thailand. Gard. Bull. Singapore 66(2): 207-214.

Mood, J.D., Veldkamp, J.F., Dey, S. \& Prince, L.M. (2014b). Nomenclatural changes in Zingiberaceae: Caulokaempferia is a superfluous name for Monolophus and Jirawongsea is reduced to Boesenbergia. Gard. Bull. Singapore 66(2): 215-231.

Mood, J.D., Hussain, A.G. \& Veldkamp, J.F. (2016a). The resurrection of Boesenbergia albosanguinea (Zingiberaceae) with a new record for Peninsular Thailand. Gard. Bull. Singapore 68(1): 109-124.

Mood, J.D., Trần, H.Đ., Veldkamp, J.F. \& Prince, L.M. (2016b). Boesenbergia siphonantha (Zingiberaceae), a new record for Thailand and Vietnam with notes on the molecular phylogeny. Gard. Bull. Singapore 68(1): 125-137. 
Posada, D. \& Crandall, K.A. (1998). ModelTest: testing the model of DNA substitution. Bioinformatics 14: 817-818.

Prince, L.M. (2010). Phylogenetic relationships and species delimitation in Canna (Cannaceae). In: Seberg, O., Pedersen, G., Barfod, A.S, \& Davis, J.I. (eds), Diversity, Phylogeny, and Evolution in the Monocotyledons: Proceedings of the Fourth International Conference on the Comparative Biology of the Monocotyledons and the Fifth International Symposium on Grass Systematics and Evolution, pp. 307-331. Aarhus: Aarhus University Press.

Rambaut, A. (1996). Se-Al Sequence Alignment Editor, ver. 2.0a11. Available from http://tree. bio.ed.ac.uk/software/seal/. Accessed 1-3 Dec. 2016.

Ronquist, F., Huelsenbeck, J. \& Teslenko, M. (2011). MrBayes: Bayesian inference of phylogeny, ver. 3.2.2. Available from the CIPRES portal. Accessed 1-3 Dec. 2016.

Sirirugsa, P. (1987). Three new species and one new combination in Boesenbergia (Zingiberaceae) from Thailand. Nordic J. Bot. 7: 421-425.

Steele, K.P. \& Vilgalys, R. (1994). Phylogenetic analysis of Polemoniaceae using nucleotide sequences of the plastid gene matK. Syst. Bot. 19: 126-142.

Swofford, D.L. (2002). PAUP*: Phylogenetic Analysis Using Parsimony (*and other methods), ver. 4.0b10. Sunderland, Massachusetts, USA: Sinauer Associates, Inc. 
Appendix 1. GenBank accession numbers for Zingiberaceae used in this study. Format: identification, plant sample number (collector and voucher number, herbarium), ITS GenBank number/trnK GenBank number.

Boesenbergia alba (K.Larsen \& R.M.Sm.) Mood \& L.M.Prince: M3253 (Mood 3253, BISH) KF982792/ KF982801

Boesenbergia bella Mood \& L.M.Prince: M3397 (Mood 16P07, BKF) KY701326/ KY701339

Boesenbergia burttii (K.Larsen \& Jenjitt.) Mood \& L.M.Prince: M12C32 (Funakoshi, H. s.n., MBK) KF982794/KF982803

Boesenbergia collinsii Mood \& L.M.Prince: M06P14 (Mood 12P171, holotype, BK) JX992751/ JX992812

Boesenbergia hamiltonii Mood et al.: M3017 (Dey, S. NU 53, holotype, CAL) JX992754/JX992815

Boesenbergia kerrii Mood et al.: M2044 (Mood \& Triboun, P. 12P170, holotype, BK) JX992756/ JX992817

Boesenbergia kingii Mood \& L.M.Prince: M3074 (Mood \& Vatcharakorn, P. 12P173, holotype, BK) JX992792/JX992829

Boesenbergia longiflora (Wall.) Kuntze: M11P48 (live material of Kress, W.J. 03-7305, US) JX992797/ JX992831

Boesenbergia maxwellii Mood et al.: M2032 (Mood \& Triboun, P. 12P172, holotype, BK) JX992800/ JX992833

Boesenbergia petiolata Sirirugsa: M3300 (Vatcharakorn, P. M3300, BKF) KY701321/ KY701334 M3332 (Mood \& Vatcharakorn, P. 3332, BKF) KY701323/ KY701336

M3501 (Mood \& Harwood, R. 3501, BKF) KY701329/ KY701342 M96P82 (Mood 96P82, BKF) KY701330/ KY701343

Boesenbergia phengklaii Mood \& Suksathan: M3307 (Mood 16P08, BKF) KY701322/ KY701335 M3349 (Mood \& Vatcharakorn, P. 3349, BKF) KY701324/ KY701337 M3478 (Mood \& Suksathan, P. 3478, BKF) KY701328/ KY701341

Boesenbergia pulcherrima (Wall.) Kuntze: M08P276 (Mood 08P276, BISH) JX992748/JX992748

Boesenbergia putiana Mood \& L.M.Prince: M3360 (Mood 16P09, BKF) KY701325/ KY701338 M3471 (Panyachan, P. s.n. QBG 20140685A, QBG). KY701327/ KY701340

Boesenbergia xiphostachya (Gagnep.) Loes.: M11C86 (Mood 11C86, BKF) KX425643/KX432277

Boesenbergia violacea (K.Larsen \& Triboun) Mood \& L.M.Prince: M12C31 (Funakoshi, H. s.n., MBK) KF982796/KF982805 
Appendix 3. Maximum likelihood phylograms of separate ITS and $\operatorname{trn} K$ data analyses for Boesenbergia petiolata and closely related taxa based on the General Time Reversible $(\mathrm{GTR}+\mathrm{I}+\mathrm{G})$ model. Branch support values are BS/PP. Taxon labels based on the recircumscribed clade including the new taxa. Top. ITS. Bottom. trnK.
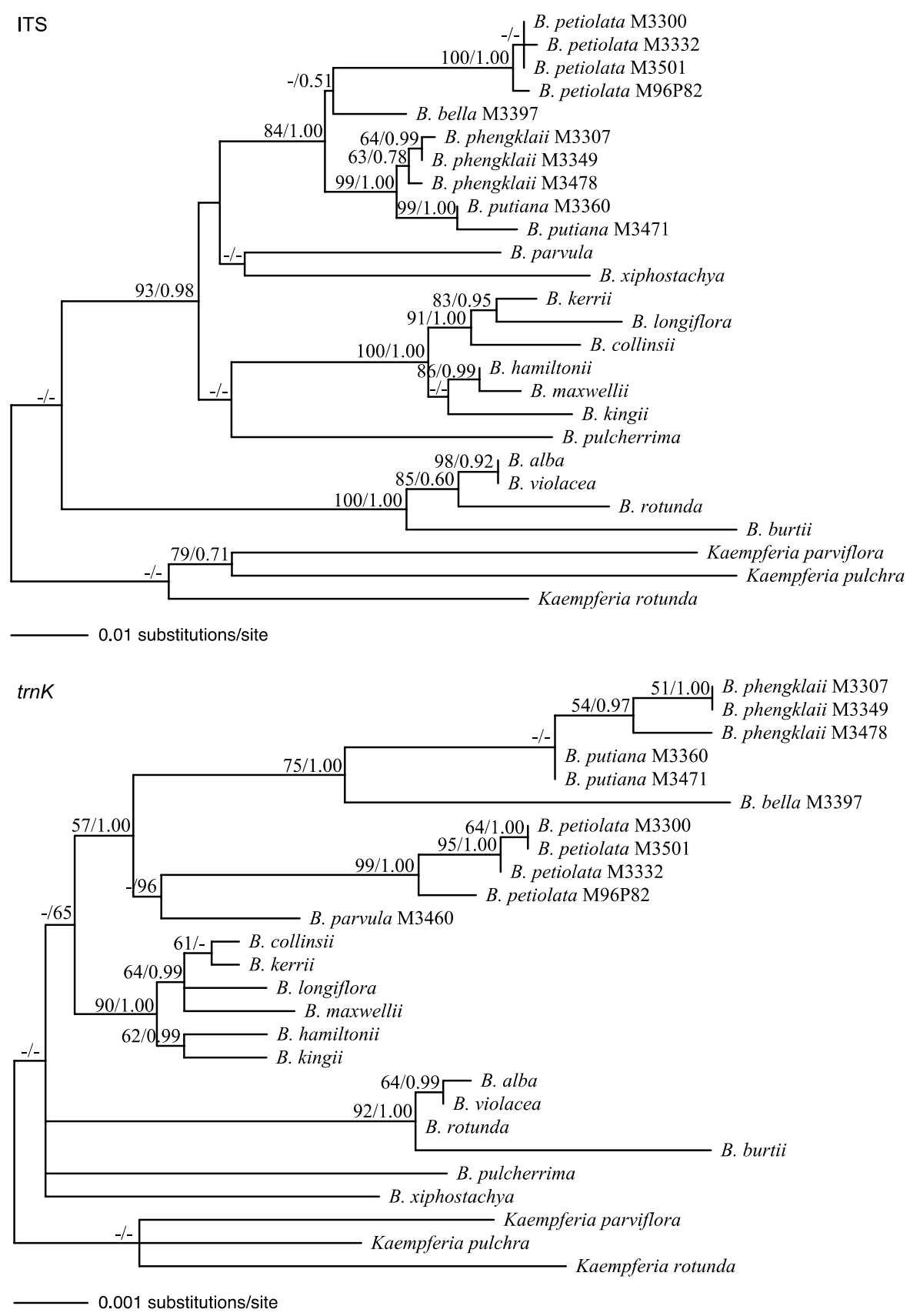